This item was submitted to Loughborough's Research Repository by the author.

Items in Figshare are protected by copyright, with all rights reserved, unless otherwise indicated.

\title{
The asymptotics of the moving contact line: cracking an old nut
}

PLEASE CITE THE PUBLISHED VERSION

https://doi.org/10.1017/jfm.2014.702

PUBLISHER

(C) Cambridge University Press (CUP)

VERSION

AM (Accepted Manuscript)

LICENCE

CC BY-NC-ND 4.0

REPOSITORY RECORD

Sibley, David, Andreas Nold, and Serafim Kalliadasis. 2015. "The Asymptotics of the Moving Contact Line: Cracking an Old Nut". Loughborough University. https://hdl.handle.net/2134/34449. 


\title{
The asymptotics of the moving contact line: Cracking an old nut
}

\author{
David N. Sibley, Andreas Nold and Serafim Kalliadasis \\ Department of Chemical Engineering, Imperial College London, London SW7 2AZ, UK
}

(Received ?; revised ?; accepted ?. - To be entered by editorial office)

For contact line motion where the full Stokes flow equations hold, full matched asymptotic solutions using slip models have been obtained for droplet spreading and more general geometries. These solutions to the singular perturbation problem in the slip length, however, all involve matching through an intermediate region that is taken to be separate from the outer-inner regions. Here, we show that the intermediate region is in fact an overlap region representing extensions of both the outer and the inner region, allowing direct matching to proceed. In particular, we investigate in detail how a previously seen result of the matching of the cubes of the free surface slope is justified in the lubrication setting. We also extend this two-region direct matching to the more general Stokes flow case, offering a new perspective on the asymptotics of the moving contact line problem.

\section{Introduction}

The moving contact line problem arises from the modelling of a system where two immiscible phases move along a solid substate using a classical hydrodynamic description with a no-slip boundary condition on the substrate (Huh \& Scriven 1971). To regularise the divergent dissipation predicted by this classical description, a wide range of possible models capturing various physical effects have been considered (see e.g. Bonn et al. (2009); Snoeijer \& Andreotti (2013); Sui et al. (2014); Sibley et al. (2014)).

One such model introduced is that of Navier-slip, and in this case the moving contact line is a singular perturbation problem. In regions away from the contact line the flow behaviour is solved at leading order satisfying the no-slip boundary condition, whereas in an inner region of $O(\lambda)$, where $\lambda$ is the nondimensional slip length, a slip boundary layer is invoked. To make the equations tractable, the contact line motion is assumed to be slow and thus there is a second, regular, perturbation in terms of $C a$, which is either the capillary number or a quantity closely related to it. Thus the problem is solved for $\lambda \ll C a \ll 1$, where (anticipating the result) $|C a \ln \lambda|=O(1)$. However, the outer and inner region solutions diverge logarithmically, with inconsistent prefactors for the logarithmic terms. It is therefore concluded that a direct matching procedure is not possible and an intermediate region is introduced, which is matched to both the outer and the inner region solutions (see e.g. the recent review of Snoeijer \& Andreotti (2013)).

In this work, we show that it is the interplay between the two expansion parameters $\lambda$ and $C a$ which leads to a breakdown of the formulations for the outer and inner region. In contrast, rearranging the $\mathrm{Ca}$-ordering of the inner and outer region approximations leads to a text-book matched asymptotic procedure, with leading order (in $\lambda$ ) solutions from both outer and inner regions being directly matched in an appropriate overlap region. In order to obtain the functional form of the outer and inner region solutions in the overlap region, one further condition is needed. We show for both Stokes flow and flow in the lubrication approximation, that this condition corresponds with the intermediate region equation derived in previous work, which we shall discuss briefly. 
A matched asymptotic description for the spreading of a thin droplet was determined by Hocking (1983), employing an intermediate region. A similar procedure to that of Hocking was considered by Eggers (2005) for a forced wetting situation, where the lubrication approximation was again made and a plate being driven into a liquid bath at constant speed. Eggers, however, directly matched the cube of the free surface slope between outer and inner regions without invoking an intermediate region. Here we give a justification as to why the direct matching procedure works. Whilst the work of Hocking (1983) concentrated on spreading (i.e. advancing contact lines), the analysis holds also for receding drops for certain values of (now negative) $\mathrm{Ca}$, as demonstrated in (Savva \& Kalliadasis 2009) with comparisons to full numerical solutions. The work of Eggers (2005) explicitly discussed receding (dewetting) contact line motion, finding a maximum (critical) value of $|C a|$ for which the analysis holds - but with no similar restriction for the advancing case. The existence of a critical $|\mathrm{Ca}|$ for receding contact lines ties with the physical picture that above a certain velocity the contact line is no longer able to remain pinned to the substrate, and rather a film of fluid is deposited.

The aforementioned work of Savva \& Kalliadasis (2009) extended the work of Hocking (1983) for spreading on ideally homogeneous surfaces to rough (topographical) substrates, where it was also noticed that the asymptotic matching procedure could be performed directly between outer and inner regions through matching of the cube of the free surface slope, with the purpose of the intermediate region to justify this procedure. This direct matching procedure has since been used to simplify the asymptotic description when considering other physical effects such as gravity (Savva \& Kalliadasis 2012), chemical heterogeneities (Vellingiri et al. 2011), and using other models in the thin-film setting (Sibley et al. 2012, 2014).

It appeared as though it was the specific structure of the solutions for the free-surface slope in these lubrication problems that allowed for the direct matching of the cubes between outer and inner regions. Here we see that indeed this is the case, but that it is a specific structure of terms not only in the first two orders of $\mathrm{Ca}$, used in all previous contact line studies, but effectively of the infinite series of the solutions in $C a$.

Similar to the three-region matching procedure in the lubrication approximation of Hocking (1983), the Stokes flow problem has been considered by Hocking \& Rivers (1982) for droplets, and later by Cox (1986) for the general motion of a contact line on a homogeneous surface. Both of these works perform the asymptotic matching with three regions. Similar results to these were also obtained by Voinov (1976). The solution for the Stokes flow problem has proven to be the cornerstone in the modelling of contact line motion, but the details of the intermediate solution are relatively complicated, and thus have probably posed an obstacle in the full understanding and further generalisation of the results for the moving contact problem. Through this work we develop a direct matching procedure for the Stokes flow problem where the cube of the slope in the thinfilm setting is a special case of a general function we derive, thus offering a reinterpretation of the matched asymptotic analysis, in particular of the so-called intermediate region.

In the following we first derive the principal result of this work in section 2. This principal result is the ability to directly match between outer and inner regions. Whilst we discuss the results in the example case of Navier-slip, we make no specific assumptions about the physics of the inner region other than that surface tension and viscous effects are included, and where microscopic effects may be neglected as we leave the inner region, moving away from the contact line. Following section 2, we then consider the specific cases of thin-film and full Stokes flow droplet spreading with slip in sections $3-4$, analysing why the matching of the cube of the free surface slope is appropriate in the lubrication approximation and how an appropriate generalisation of this works 
for the full Stokes flow situation. Finally, we give conclusions in section 5, including a discussion on the applicability of our results for positive and negative $C a$ (for advancing and receding contact lines, respectively), and applicability for other contact line models including more complex physics.

\section{The principal result}

Our principal result is that matching between outer and inner regions may proceed directly for moving contact line problems. There is an overlap region, however, formulating the solutions as a regular perturbation in $C a$ and only retaining a finite number of terms (as is commonly done) leads to the breakdown of this overlap region. Regrouping the series of solutions by isolating the terms that cause this breakdown, and thus incorporating this infinite series of non-negligible terms into our solution, allows the overlap region to be retained.

To provide a guide to the asymptotic regions and procedure, we show in figure 1 a comparison between full numerical and asymptotic solutions of the forced wetting problem in the thin-film regime (for more details, see section 3.1 and Appendix A). In particular, in figure 1(a) we show the extent of applicability of outer and inner regions (calculated analytically), and how the overlap breaks down when the $C a$-series of solutions is truncated. As a greater number of terms in the $C a$ perturbation are retained, the extent of the breakdown of the overlap region reduces. In figure 1(b) we again compare the analytic outer region solutions, but to the full (infinite-order in $\mathrm{Ca}$ ) numerical inner region solution, computed with a cut-off at a distance $x / \lambda=L$. Three full numerical inner region solutions are shown due to the numerical implementation in the finite domain $x / \lambda=[0, L]$, where $L \rightarrow \infty$ (more details in Appendix A). Even when only having solutions up to (and including) $O\left(\mathrm{Ca}^{2}\right)$ in the outer solution, one can observe the overlap between outer and inner regions.

In all problems for slow motion of contact lines, including the thin-film and Stokes regimes discussed in Cox (1986); Hocking \& Rivers (1982); Eggers (2005); Hocking (1983), the behaviour of the free-surface slope in outer (slip is negligible) and inner (slip is significant) regions can be written in a general form as

$$
\begin{aligned}
h_{\text {out }}^{\prime}(x) & \sim \theta_{\text {out }}+C a\left[f\left(\theta_{\text {out }}\right) \ln x+C_{\text {out }}\right]+O\left(\lambda, x, C a^{2}, C a x \ln x\right), \quad \text { as } x \rightarrow 0, \\
h_{\text {in }}^{\prime}(x) & \sim \theta_{\text {in }}+C a\left[f\left(\theta_{\text {in }}\right) \ln \frac{x}{\lambda}+C_{\text {in }}\right]+O\left(\lambda, C a^{2}, \frac{\lambda C a}{x} \ln \frac{x}{\lambda}\right), \quad \text { as } \frac{x}{\lambda} \rightarrow \infty .
\end{aligned}
$$

Here $h^{\prime}$ denotes the slope of the free surface, the spatial variable has been denoted $x$, with $x=0$ being the location of the contact line (and taken $x \geqslant 0$ ), and $\lambda \ll C a \ll 1$, where $C a$ represents the spreading rate or capillary number, and $\lambda$ is the slip length. The unspecified parameters $\left\{\theta_{\text {out }}, \theta_{\text {in }}, C_{\text {out }}, C_{\text {in }}\right\}$ and the function $f$ are different for each specific situation being considered, but could depend on quantities such as the viscosity ratio of the fluids in contact or the droplet radius (if a droplet is considered).

The observation is usually made that in (2.1) the logarithmic terms in $x$ do not match, because of the different prefactors $f\left(\theta_{\text {out }}\right)$ and $f\left(\theta_{\text {in }}\right)$, with two different corollaries offered. Either as these terms do not match, an intermediate region must be necessary to bridge the solutions (see also Cox (1986); Hocking (1983); Hocking \& Rivers (1982)); or as these terms do not match, the appropriate variable for matching is not $h^{\prime}$, but that there may be a different variable that can directly match (in the thin-film situation, this being identified as $h^{\prime 3}$, see also Eggers (2005); Savva \& Kalliadasis (2009)). As we will see, whilst these both work, neither gives the full story. 
(a)

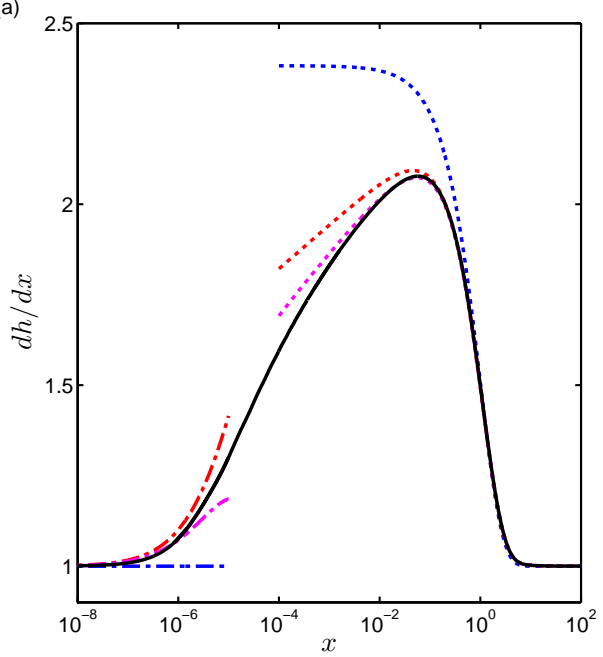

(b)

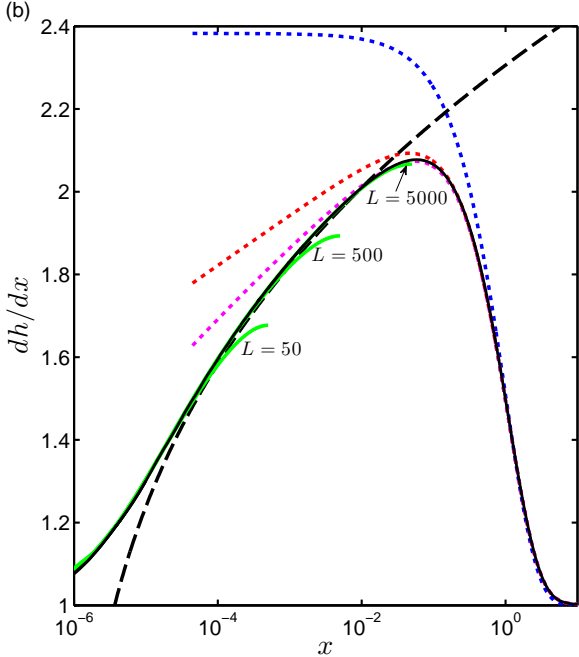

FiguRE 1. Comparison of full numerical and asymptotic solutions of the forced wetting problem in the thin-film regime. The figure is plotted for parameters $\lambda=10^{-5}$ and $C a=0.3$, such as defined in section 3.1, with the apparent (rescaled) contact angle calculated as $\theta_{\text {out }}=2.38$. (a) shows the convergence of outer and inner asymptotic solutions to the full solution, with the first three orders in $C a$ given, and where the solid line represents the full numerical solution of (3.1). The dotted and dash-dotted lines represent different levels of the expansion of the outer and inner solution in terms of $C a$, respectively. Blue, red and magenta correspond to the leading, first and second order solutions in $\mathrm{Ca}$. (b) shows the overlap between outer and inner regions. Here the outer region solutions from (a) are repeated, but compared to full (infinite-order in $\mathrm{Ca}$ ) numerical inner region solutions. Three of these numerical solutions are shown as green solid lines to see numerical convergence due to the numerical implementation in a finite domain $x / \lambda=[0, L]$, where $L \rightarrow \infty$, more details in Appendix A. The dashed line is the intermediate solution of the problem, which shows the limiting behaviour of outer and inner regions as they extend to 0 and $\infty$, respectively.

The critical feature to understand of the expansions (2.1) is that it is a two-parameter expansion in $\lambda$ and then $\mathrm{Ca}$. All of the terms retained throughout this work are at leading order in the singular perturbation of $\lambda$, hence corrections of $O(\lambda)$. In (2.1), two orders in the $C a$ perturbation, $O\left(C a^{0}\right)$ and $O\left(C a^{1}\right)$ have been retained, with terms of $O\left(C a^{2}\right)$ neglected. When $x$ takes values that are within the regions of applicability of (2.1), and when also $C a \ln x$ and $C a \ln (x / \lambda)$ are genuinely small, the $C a$-ordering of the terms in (2.1) is satisfied. Essentially, the region of validity of the outer and inner regions, if the $\mathrm{Ca}$-series is truncated, is limited by

$$
|C a \ln x| \ll 1 \Rightarrow x \gg \mathrm{e}^{-1 /|C a|} \quad \text { and } \quad|C a \ln (x / \lambda)| \ll 1 \Rightarrow x \ll \lambda \mathrm{e}^{1 /|C a|},
$$

respectively. Thus, there is only an overlap between outer and inner regions in this truncated scenario if $|C a \ln \lambda| \ll 2$.

The difficulties arise when this condition is not satisfied. In this case, as $x \rightarrow 0$ from the outer region, terms of $O(|C a \ln x|)$ become $O(1)$ before the region of validity of the inner region expansion is approached. The same holds for terms of $O(|C a \ln (x / \lambda)|)$ for $x / \lambda \rightarrow \infty$, coming from the inner region. This means that neglecting terms of $O\left(C a^{2}\right)$ in the expansions (2.1) is not possible if terms such as $(C a \ln x)^{2}$ occur (which they do), and indeed terms of $(C a \ln x)^{n}$ for any positive integer $n$, may not be neglected. Let us define an overlap region where $|C a \ln x|=O(1)$. Using this, we thus return to the outer 
expansion (2.1a), and instead consider an expansion of the form

$$
h_{\text {out }}^{\prime}(x) \sim \sum_{n=0}^{\infty} a_{n}(C a \ln x)^{n}+C a \sum_{n=0}^{\infty} b_{n}(C a \ln x)^{n}+O\left(C a^{2}, x, C a x \ln x, \lambda\right), \quad \text { as } x \rightarrow 0,
$$

where we retain the first two summations in an expansion in $\mathrm{Ca}$. Here two terms have been retained as this is related to the order of accuracy we wish to obtain in our final result, achieving a correction of $O\left(\mathrm{Ca}^{2}\right)$.

In our context, considering (2.3) as an extension of (2.1a) we thus have

$$
\begin{aligned}
h_{\text {out }}^{\prime}(x) \sim \theta_{\text {out }} & +C a\left[f\left(\theta_{\text {out }}\right) \ln x+C_{\text {out }}\right]+\sum_{n=2}^{\infty} a_{n}(C a \ln x)^{n}+\sum_{n=1}^{\infty} C a b_{n}(C a \ln x)^{n} \\
+ & O\left(C a^{2}, x, C a x \ln x, \lambda\right), \quad \text { as } x \rightarrow 0,
\end{aligned}
$$

where now all of the neglected terms will always be negligible in comparison to those retained (for $\lambda \ll x \ll 1$ ). We may now rewrite expansion (2.4) in terms of the variable

$$
z=C a \ln x
$$

which is an $O(1)$ quantity in the overlap region, and where we collect terms to obtain

$$
\begin{array}{ll} 
& h_{\text {out }}^{\prime}(z) \sim T_{0}(z)+C a T_{1}(z)+O\left(C a^{2}, \mathrm{e}^{z / C a}, z \mathrm{e}^{z / C a}, \lambda\right), \quad \text { as } x \rightarrow 0, \\
\text { where } \quad & T_{0}(z)=\theta_{\text {out }}+f\left(\theta_{\text {out }}\right) z+\sum_{n=2}^{\infty} a_{n} z^{n}, \quad T_{1}(z)=C_{\text {out }}+\sum_{n=1}^{\infty} b_{n} z^{n} .
\end{array}
$$

In order to obtain forms of $T_{0}(z)$ and $T_{1}(z)$, additional information is needed. Either all non-negligible terms in (2.4) are determined through e.g. an iterative procedure to find solutions at higher orders of $\mathrm{Ca}$, or alternatively, they are obtained via a method described in the remainder of this section.

As suggested by the behaviour of $d_{z} h_{\text {out }}^{\prime}$ in (2.6), we determine a condition to hold for the overlap region of

$$
\frac{x}{C a} \frac{d h^{\prime}}{d x}=f\left(h^{\prime}\right)+O\left(C a^{2} ; \frac{x}{C a}, x \ln x ; \frac{\lambda}{x} \ln \frac{x}{\lambda}\right) .
$$

This equation corresponds to the physical balance of viscous and surface tension effects, defining the mesoscopic hydrodynamic regime such as described by Snoeijer \& Andreotti (2013). We may motivate the expression as these contributions affect the free surface through the normal stress boundary condition, which leads to (2.7) in this context. We must, however, verify that (2.7) indeed holds for each individual contact line geometry/case separately.

As we show in the following sections, (2.7) is equivalent to the intermediate region equations found in the literature. Here, we instead note that it holds for both outer and inner regions, and throughout their overlap. By considering the left hand side, which corresponds to any of

$$
\frac{d h^{\prime}}{d z}=\frac{x}{C a} \frac{d h_{\mathrm{out}}^{\prime}}{d x}=\frac{(x / \lambda)}{C a} \frac{d h_{\mathrm{in}}^{\prime}}{d(x / \lambda)}
$$

we see that the terms retained in (2.7) are of comparable order, and the quantities neglected are small in both the outer region as $x \rightarrow 0$ and the inner region as $(x / \lambda) \rightarrow \infty$. We conclude that equation (2.7) describes the limiting behaviour of both outer and inner region equations, and consequently it describes both the limiting behaviour of $h_{\mathrm{in}}^{\prime}$ 
and $h_{\text {out }}^{\prime}$. We hence see that what is usually known as the intermediate region is in fact an overlap region and that the apparent breakdown of the outer and inner region solutions is an artifact due to the two-parameter expansion in the slip length $\lambda$ and the nondimensional contact line speed $\mathrm{Ca}$. For the outer region slope, (2.7) is

$$
\frac{d h_{\text {out }}^{\prime}}{d z}=f\left(h_{\text {out }}^{\prime}\right)+O\left(C a^{2}, C a^{-1} \mathrm{e}^{z / C a}\right),
$$

where the other correction $O\left(z \mathrm{e}^{z / C a} \mathrm{Ca}^{-1}\right)$ is no longer included as $|z|=O(1)$. Let us now obtain the limiting behaviour of the outer solution by solving (2.9) with a boundary condition suggested by (2.6) at $\left|z_{0}\right|=O\left(C a^{2}\right), z_{0}<0$ of

$$
h_{\text {out }}^{\prime}\left(z_{0}\right)=\theta_{\text {out }}+C a C_{\text {out }}+O\left(C a^{2}\right) .
$$

Let us now use (2.6) in (2.9), giving

$$
d_{z} T_{0}(z)=f\left(T_{0}(z)\right), \quad d_{z} T_{1}(z)=f^{\prime}\left(T_{0}(z)\right) T_{1}(z)
$$

where we note that these expressions now contain all terms $(C a \ln x)^{n}$ and $C a(C a \ln x)^{n}$ as they are assembled in $T_{0}$, and $T_{1}$. This thus means that through solving (2.11), we are able to determine the sum of the prefactors of all terms $(C a \ln x)^{n}$, and $C a(C a \ln x)^{n}$. The leading order of (2.11) may be solved to find

$$
T_{0}(z)=G^{-1}\left(z+A_{c}\right), \quad \text { where } \quad G\left(T_{0}\right)=\int_{0}^{T_{0}} \frac{1}{f(a)} \mathrm{d} a,
$$

where $A_{c}$ is a constant of integration. The next-to-leading order equation is then

$$
T_{1}(z)=B_{c} f\left(T_{0}(z)\right)=B_{c} f\left(G^{-1}\left(z+A_{c}\right)\right)
$$

such that

$$
\begin{aligned}
h_{\mathrm{out}}^{\prime} & \sim G^{-1}\left(z+A_{c}\right)+C a B_{c} f\left(G^{-1}\left(z+A_{c}\right)\right)+O\left(C a^{2}, \lambda, e^{z / C a}\right) \\
& \sim G^{-1}\left(z+A_{c}+C a B_{c}\right)+O\left(C a^{2}, \lambda, e^{z / C a}\right),
\end{aligned}
$$

given that the $C a$-ordering of (2.6) holds and employing (2.12), noting in general that $\left(G^{-1}\right)^{\prime}(a)=1 /\left[G^{\prime}\left(G^{-1}(a)\right)\right]$, so that here $\left(G^{-1}\right)^{\prime}(T)=f\left(G^{-1}(T)\right)$. By enforcing boundary condition (2.10) we thus determine $A_{c}=G\left(\theta_{\text {out }}\right)$, and $B_{c}=C_{\text {out }} / f\left(\theta_{\text {out }}\right)$. The full asymptotic behaviour of the outer solution as $x \rightarrow 0$ is then

$$
h_{\text {out }}^{\prime} \sim G^{-1}\left(C a \ln x+G\left(\theta_{\text {out }}\right)+C a \frac{C_{\text {out }}}{f\left(\theta_{\text {out }}\right)}\right)+O\left(C a^{2}, \lambda, x\right) .
$$

Crucially, an analogous procedure can be applied to the limiting behaviour of the inner region, such that

$$
h_{\mathrm{in}}^{\prime} \sim G^{-1}\left(C a \ln \left(\frac{x}{\lambda}\right)+G\left(\theta_{\mathrm{in}}\right)+C a \frac{C_{\mathrm{in}}}{f\left(\theta_{\mathrm{in}}\right)}\right)+O\left(C a^{2}, \lambda, \frac{\lambda}{x}\right) .
$$

2.1. The principal result of overlap and direct matching

Comparing (2.15) and (2.16), it can now be seen that the outer and inner solution coincide in the full overlap region, with matching through

$$
\lim _{x / \lambda \rightarrow \infty} h_{\text {in }}^{\prime}=\lim _{x \rightarrow 0} h_{\text {out }}^{\prime},
$$

giving the result

$$
G\left(\theta_{\text {out }}\right)-G\left(\theta_{\text {in }}\right)=C a\left[-\ln \lambda+\frac{C_{\text {in }}}{f\left(\theta_{\text {in }}\right)}-\frac{C_{\text {out }}}{f\left(\theta_{\text {out }}\right)}\right]+O\left(C a^{2}\right),
$$


and being the same result as the matched asymptotic derivations for Stokes and thin-film problems discussed in the introduction, which employ a separate intermediate region. We note that $C a$ in (2.18) may be be positive or negative depending on whether advancing or receding flows are considered, respectively, but that for the receding flows the result holds only up to a critical value of $|C a|$ when it is negative. This is as the validity of (2.18) depends on (2.7), which will only hold up to this critical value of $|C a|$ for receding flows. Further discussion will be given in the conclusions in section 5 .

\subsection{Corollary of the direct matching}

Now that we have justified the direct matching procedure provided (2.7) holds, we see that the generalisation of 'matching the cube of the free surface slope' corresponds in fact to matching $G\left(h^{\prime}\right)$. Thus we may apply $G$ onto the expansion (2.1)

$$
\begin{aligned}
G\left(h_{\text {out }}^{\prime}\right) & =\int_{0}^{\theta_{\text {out }}+C a\left[f\left(\theta_{\text {out }}\right) \ln x+C_{\text {out }}\right]+\ldots} \frac{\mathrm{d} A}{f(A)} \sim G\left(\theta_{\text {out }}\right)+C a\left[\ln x+\frac{C_{\text {out }}}{f\left(\theta_{\text {out }}\right)}\right]+O\left(C a^{2}\right), \\
G\left(h_{\text {in }}^{\prime}\right) & =\int_{0}^{\theta_{\text {in }}+C a\left[f\left(\theta_{\text {in }}\right) \ln \frac{x}{\lambda}+C_{\text {in }}\right]+\ldots} \frac{\mathrm{d} A}{f(A)} \sim G\left(\theta_{\text {in }}\right)+C a\left[\ln \frac{x}{\lambda}+\frac{C_{\text {in }}}{f\left(\theta_{\text {in }}\right)}\right]+O\left(C a^{2}\right),
\end{aligned}
$$

where it is now seen that the logarithmic terms in $x$ match (which we knew by construction), and matching the remaining terms gives the result (2.18). In the following, we show that the intermediate region equations derived in the literature to solve moving contact line problems are in fact equivalent to (2.7).

\section{Thin-film cases}

The situation that motivated the present work was that of the spreading of a thin two-dimensional droplet, as it was shown that matching could be performed on the cube of the free surface slope (Savva \& Kalliadasis 2009). Additionally, direct matching was also performed between outer and inner regions with this cube in the work of Eggers (2005) on forced wetting, as discussed in the introduction. In the following we will show that the intermediate region equations are indeed equivalent to (2.7), hence allowing for an overlap region and the direct matching of the previous section to be employed.

\subsection{Forced wetting}

Consider the case of forced wetting where solid substrate is plunged into a liquid bath at small plate angles, as studied e.g. by Eggers (2005). We consider the typical length scale to be the capillary length $L=\sqrt{\sigma /(\rho g)}$, with the Bond number then $\rho g L^{2} / \sigma=1$, and a small ratio of vertical vs longitudinal length scale $\varepsilon=H / L=\theta_{e}$, set to be the equilibrium contact angle $\theta_{e} \ll 1$. The evolution equation for the height $h$ then transforms to

$$
-\frac{C a}{h^{2}+\lambda h}=h^{\prime \prime \prime}-h^{\prime}+\theta
$$

where for simplicity, we have set the dimensionless slip length to $\lambda=3 \lambda_{\operatorname{dim}} / L$, the capillary number to $3(U \mu / \sigma) / \theta_{e}^{3}$, and $\theta$ represents the normalized plate angle. A rescaling with $\lambda$ leads to the inner region behaviour

$$
h_{\text {in }}^{\prime}(x) \sim 1+C a[\ln (x / \lambda)+1]+O\left(C a^{2}\right), \quad \text { as } x / \lambda \rightarrow \infty,
$$

which corresponds to equation $(2.1 b)$, with $\theta_{\text {in }}=C_{\text {in }}=1$. The outer region behaviour is known to be

$$
h_{\text {out }}^{\prime}=\theta_{\text {out }}+C a \theta_{\text {out }}^{-2}[\ln x+C]+O\left(x, C a^{2}\right), \quad \text { as } x \rightarrow 0,
$$


where $C$ depends on $\theta_{\text {out }}$ and is given explicitly in Eggers (2005). Let us now consider what is known in the literature as the intermediate region (although Eggers (2005) did not consider this in his work). This is obtained by defining intermediate variables, being equivalent to those used in other situations by Hocking (1983); Hocking \& Rivers (1982); Cox (1986), as

$$
S(z)=h(x) / x, \quad z=C a \ln x,
$$

such that equation (3.1) transforms to

$$
1=S\left[S+\lambda \mathrm{e}^{-z / C a}\right]\left[S^{\prime}-C a^{2} S^{\prime \prime \prime}+\frac{\mathrm{e}^{2 z / C a}}{C a}\left(S-\theta+C a S^{\prime}\right)\right] .
$$

We can reduce this equation to one more closely resembling (2.7) provided $S^{\prime \prime \prime}=O\left(S^{\prime}\right)$. The restriction $S^{\prime \prime \prime}=O\left(S^{\prime}\right)$ holds for advancing contact lines where $C a>0$, but for receding cases above a critical value of $|C a|$ it breaks down. This manifests itself here as above the critical value the matching behaviour of the inner region gives a constant curvature, with then $h \sim x^{2} / \lambda$. In the variables of (3.4) this suggests $S \sim \lambda^{-1} \mathrm{e}^{z / C a}$, and hence we may not neglect the term involving the third derivative - given it has leading order behaviour $S^{\prime \prime \prime} \sim \mathrm{Ca}^{-2} S^{\prime}$.

Additionally, above the critical value, the breakdown of assumption $S^{\prime \prime \prime}=O\left(S^{\prime}\right)$ also corresponds to the collapse of expansion $(2.1 b)$ in the inner region due to an additional correction term $x / \lambda$ and our direct matching procedure of section 2 only applies when expansions (2.1) hold. Considering now the regime below the critical value for $\mathrm{Ca}<0$, and for $C a>0$, equation (3.5) simplifies to

$$
1=S^{2} S^{\prime}+O\left(C a^{2}, \lambda, \frac{\mathrm{e}^{2 z / C a}}{C a}\right)
$$

or

$$
S^{\prime}(z)=f_{\mathrm{tf}}(S)+O\left(C a^{2}, \lambda, \frac{\mathrm{e}^{2 z / C a}}{C a}\right) \quad \text { with } \quad f_{\mathrm{tf}}(S)=\frac{1}{S^{2}},
$$

noting that as $x=\mathrm{e}^{z / C a} \ll 1$ the neglected exponential terms are indeed small (i.e. for $C a>0, z<0$ in this region). Continuing in the regimes where (3.7) holds, from (3.4) we have that

$$
\frac{d h}{d x}=S+C a S^{\prime}, \quad \text { and thus } \frac{d}{d z}\left(\frac{d h}{d x}\right)=S^{\prime}+C a S^{\prime \prime},
$$

and using (3.7) and $S^{\prime \prime}(z)=f_{\mathrm{tf}}^{\prime}(S) S^{\prime}+O\left(C a^{2}, \lambda, C a^{-1} \mathrm{e}^{2 z / C a}\right)$ then

$$
\begin{aligned}
\frac{d}{d z}\left(\frac{d h}{d x}\right) & =f_{\mathrm{tf}}(S)+C a f_{\mathrm{tf}}^{\prime}(S) S^{\prime}+O\left(C a^{2}, \lambda, C a^{-1} \mathrm{e}^{2 z / C a}\right) \\
& =f_{\mathrm{tf}}\left(S+C a S^{\prime}\right)+O\left(C a^{2}, \lambda, C a^{-1} \mathrm{e}^{2 z / C a}\right) \\
& =f_{\mathrm{tf}}\left(\frac{d h}{d x}\right)+O\left(C a^{2}, \lambda, \frac{\mathrm{e}^{2 z / C a}}{C a}\right)
\end{aligned}
$$

which is equivalent to (2.7). Thus, if similar intermediate regions are shown to be of the form (3.7) with an $O\left(\mathrm{Ca}^{2}\right)$ correction, then this is equivalent to having (2.7) hold. By contrast, if there is an $\mathrm{O}(\mathrm{Ca})$ correction in an equation such as (3.7) formulated in an intermediate region, then (2.7) will hold only up to $O(\mathrm{Ca})$, and thus the final result (2.18) only holds for coefficients $O(1)$ and $O(C a \ln \lambda$ ) (but not including $O(C a)$ ). We conclude 
that in the thin-film case, the map $G$ is defined by

$$
G_{\mathrm{tf}}\left(h^{\prime}\right)=\int_{0}^{h^{\prime}} \frac{1}{f_{\mathrm{tf}}(a)} d a=\frac{h^{\prime 3}}{3}
$$

giving a clear indication as to why matching the cube of the free surface slope is the correct procedure in this case. In figure 1, a comparison of numerical and asymptotic results is shown.

\subsection{Thin-film droplet spreading/receding}

Analysis of thin-film droplet spreading was performed by Hocking (1983) on homogeneous substrates, but has been extended to consider a range of different complicating physics such as gravity (Savva \& Kalliadasis 2012), chemical and substrate heterogeneities (Savva \& Kalliadasis 2009; Vellingiri et al. 2011). We will briefly revisit symmetric two-dimensional spreading/receding on a homogeneous surface with negligible gravitational effects to see how direct matching proceeds.

In the inner region the behaviour is the same as given for the forced wetting case in (3.2), but with $C a=\dot{a}$ ( $\dot{a}$ being the spreading/receding rate of the droplet, and $a(t)$ the location of the contact line), and $x=a-\hat{x}$ where $\hat{x}$ is the spatial coordinate along the substrate. The intermediate region equation given in (3.19) of Hocking (1983) and similarly in (52) of Savva \& Kalliadasis (2009), corresponds with the intermediate region equation in the forced wetting case (3.7), which we have already shown to be equivalent to the overlap condition (2.7) here. In the outer region, where slip is negligible, it is possible to find the behaviour in a quasistatic expansion $(\lambda \ll|\dot{a}| \ll 1)$ for the first three orders (following the analysis of Sibley et al. (2012)). We find as $\hat{x} \rightarrow a$ that

$$
-\frac{\partial h}{\partial \hat{x}} \sim \frac{3}{a^{2}}+\frac{\dot{a} a^{4}}{9} \ln \left[\frac{\mathrm{e}^{3} x}{2 a}\right]-\frac{a^{10} \dot{a}^{2}}{243}\left\{(\ln x)^{2}-2 \ln \left[\frac{2 a}{\mathrm{e}^{3}}\right] \ln x+O(1)\right\} .
$$

Here then $\theta_{\text {out }}=3 a^{-2}, f_{\mathrm{tf}}(\theta)=\theta^{-2}, C_{\text {out }}=a^{4} \ln \left[\mathrm{e}^{3} /(2 a)\right] / 9$. Thus, we have $G_{\mathrm{tf}}(\theta)=$ $\theta^{3} / 3$, and the matching condition (2.18) then corresponds to $3 \dot{a} a^{6} \ln \left[\lambda \mathrm{e}^{2} /(2 a)\right]=a^{6}-27$.

\subsection{Dewetting corner tip}

A further case related to droplet motion of interest to discuss is that of the behaviour of the receding corner tip of a droplet, as studied by Peters et al. (2009). The authors took an inner region Cox-Voinov law, the thin-film equivalent of (2.16), and matched it to the appropriate outer region behaviour again directly using the cube of the free-surface slope (in a similar way to the work of Eggers, discussed in the previous subsection). The authors thus obtained a version of the general result (2.18), being

$$
\theta_{\text {out }}^{3}-1=3 C a\left[-\ln \lambda+\ln \left(\frac{\beta R}{\phi^{2}}\right)\right] \text {, }
$$

where $R$ is the droplet tip radius, $\phi$ is the opening angle of the leading order outer solution (modelled approximately by a hyperbola), $\beta$ is used as a 'fit parameter', and we reiterate that in our notation for dewetting $C a=3(\mu U / \sigma) / \theta_{e}^{3}<0$ (noting the factor $3 / \theta_{e}^{3}$ as compared to Peters et al.). Peters et al. (2009) finally suggest that $\theta_{\text {out }} \ll 1$ in the corner tip regime, and thus obtain the scaling law $R=\ell \mathrm{e}^{1 /(3 C a)}$, where $\ell=\lambda \phi^{2} / \beta$. Concluding then, the present analysis of section 2 justifies why matching the cube of the free surface slope was an appropriate method for Peters et al. (2009). 


\section{Stokes flow cases}

In comparison to the asymptotic analysis in the thin-film setting, when considering full Stokes flow the analysis becomes substantially more involved, but the general features of outer (insignificant slip) and inner (slip important) regions remain. In this section for notational convenience we still use $h^{\prime}$, however this denotes the angle of inclination of the free-surface.

\subsection{Matching for the Cox (1986) result}

We consider the Cox (1986) analysis in light of our direct matching procedure, where the outer and inner matching behaviours were found respectively as

$$
\begin{aligned}
h_{\text {out }}^{\prime} & \sim \theta_{\text {out }}+C a\left[f_{\mathrm{C}}\left(\theta_{\text {out }}\right) \ln r+Q_{o}^{*}+\ldots\right]+\ldots, & & \text { as } r \rightarrow 0, \\
h_{\text {in }}^{\prime} & \sim \theta_{\text {in }}+C a\left[f_{\mathrm{C}}\left(\theta_{\text {in }}\right) \ln (r / \lambda)+Q_{i}^{*}+\ldots\right]+\ldots, & & \text { as } r / \lambda \rightarrow \infty,
\end{aligned}
$$

where $f_{\mathrm{C}}(\theta)$ is given by

$$
f_{\mathrm{C}}(\theta)=\frac{2 \sin \theta\left[L^{2}\left(\theta^{2}-\sin ^{2} \theta\right)+2 L\left(\theta(\pi-\theta)+\sin ^{2} \theta\right)+(\pi-\theta)^{2}-\sin ^{2} \theta\right]}{L\left(\theta^{2}-\sin ^{2} \theta\right)(\pi-\theta+\sin \theta \cos \theta)+\left((\pi-\theta)^{2}-\sin ^{2} \theta\right)(\theta-\sin \theta \cos \theta)},
$$

which arises from the solution of the Biharmonic equation for the stream function for two fluids of viscosities $\mu_{A}$ and $\mu_{B}$ (with viscosity ratio $L=\mu_{B} / \mu_{A}$ ), applied in the normal stress condition at leading order (Cox 1986). Here, in keeping with the notation of Cox (1986), $r$ is the radial distance from the contact line, $Q_{o}^{*}$ and $Q_{i}^{*}$ are unspecified constants of integration which depend on the specific problem to be considered, and $C a \ll 1$ is the capillary number, where Cox already identified that receding motion is found by mapping $C a \mapsto-C a$. Note that we use $\lambda$ to be the slip length here in comparison to Cox's use of $\epsilon$, to avoid confusion with the previous sections.

In general, one could solve the Stokes equation with the normal stress boundary condition for the leading order terms (in $\ln r$ ) to all $C a$-number orders, obtain all terms in (2.6) and thus the form of $T_{0}$ and $T_{1}$. However, as this is rather involved, one can alternatively show the validity of the overlap region equation (2.7). Cox (1986) derives equation (7.9) in his manuscript for a variable $\tilde{\beta}_{0}$, it being

$$
d_{z} \tilde{\beta}_{0}=f_{\mathrm{C}}\left(\tilde{\beta}_{0}\right)+O\left(C a^{2}\right)
$$

where for clarity we have denoted the intermediate variable by $z=C a \ln r$ instead $\tilde{x}$, the notation used by Cox. $\tilde{\beta}_{0}$ is related to the slope $\theta$ of the interface as defined in equation (7.12) of his manuscript, by $\theta=\tilde{\beta}_{0}+C a d_{z} \tilde{\beta}_{0}+O\left(C a^{2}\right)$. It may thus be shown that

$$
d_{z} \theta=f_{\mathrm{C}}(\theta)+O\left(C a^{2}\right),
$$

showing the equivalence to the overlap equation (2.7). We may thus use the same procedure as discussed in section 2 , and we find that

$$
G_{\mathrm{C}}\left(\theta_{\text {out }}\right)-G_{\mathrm{C}}\left(\theta_{\text {in }}\right)=C a\left[-\ln \lambda+Q_{i}^{*} / f_{\mathrm{C}}\left(\theta_{\text {in }}\right)-Q_{o}^{*} / f_{\mathrm{C}}\left(\theta_{\text {out }}\right)\right]+O\left(C a^{2}\right) .
$$

In contrast, we note that the case of inertial contact line motion investigated by Cox (1998) (and recently analysed numerically by Ding \& Spelt (2007)) will not satisfy (2.7), e.g. as the intermediate equation equivalent to (3.7) has a correction of $O(\mathrm{Ca})$ in this case - see equations (6.35)-(6.38) of (Cox 1998). In this inertial case then without further information, our direct matching procedure can only find a result including terms up to $O(C a \ln \lambda)$. The result is $G_{\mathrm{CI}}\left(\theta_{\text {out }}\right)=G_{\mathrm{CI}}\left(\theta_{\text {in }}\right)-C a \ln \lambda$, where $G_{\mathrm{CI}}(\theta)=4 \alpha^{*-2}(\theta-\sin \theta)$, and $f_{\mathrm{CI}}(\theta)=\alpha^{* 2} \csc ^{2}(\theta / 2) / 8$ (where $\left.\alpha^{*}=1.61605\right)$. See (Cox 1998) for further details, and (7.6) there to see the agreement with this result. 


\subsection{Hocking and Rivers}

For a droplet in the Stokes regime the expansions for the outer and inner regions as $x \rightarrow 0$ and $x / \lambda \rightarrow \infty$, respectively, are taken from the analysis of Hocking \& Rivers (1982):

$$
\begin{aligned}
h_{\text {out }}^{\prime} & \sim \theta_{\text {out }}+\epsilon\left[\sin \theta_{\text {out }} /\left(\theta_{\text {out }}-\sin \theta_{\text {out }} \cos \theta_{\text {out }}\right)\right]\left[\ln (\mathrm{e} x / a)+Q_{0}\left(\theta_{\text {out }}\right)\right], \\
h_{\text {in }}^{\prime} & \sim \theta_{\text {in }}+\epsilon\left[\sin \theta_{\text {in }} /\left(\theta_{\text {in }}-\sin \theta_{\text {in }} \cos \theta_{\text {in }}\right)\right]\left[\ln (\mathrm{e} x / \lambda)+Q_{1}\left(\theta_{\text {in }}\right)\right],
\end{aligned}
$$

where $\epsilon=2 \dot{a} \mu / \sigma$ is the small spreading parameter in this problem. Here additional variables of note are $x$, which is now an arc length measured from the edge of the droplet, and $Q_{0}\left(\theta_{\text {out }}\right)$ and $Q_{1}\left(\theta_{\text {in }}\right)$ are functions of the apparent and static contact angles (see Hocking \& Rivers (1982) for further details). Note that we have replaced $\alpha, \alpha_{s}$ in Hocking \& Rivers (1982) notation by $\theta_{\text {out }}, \theta_{\text {in }}$ for the sake of consistency here. Also, $\mu$ and $\sigma$ are the viscosity and surface tension of the droplet, respectively, and $a$ is now the dimensional droplet radius (with $\dot{a}$ the dimensional spreading rate).

We see, as for the previous examples, that the logarithmic terms do not balance, but that once again both behaviours have the general form in (2.1). Unlike the thin-film regime, where $f_{\mathrm{tf}}(A)=1 / A^{2}$, here the form is

$$
f_{\mathrm{HR}}(A)=\sin A /(A-\sin A \cos A),
$$

which was also shown to be an important function for contact line motion by Voinov (1976); Snoeijer (2006). This function is also obtained (up to a multiplicative constant) from Cox's function in (4.2) when $L=0$. The equivalent to the overlap region equation (2.7) is given in equation (5.2) of Hocking \& Rivers (1982). This shows that matching will be achieved with the function $G$ being

$$
G_{\mathrm{HR}}\left(h^{\prime}\right)=\int_{0}^{h^{\prime}} \frac{\mathrm{d} \zeta}{f_{\mathrm{HR}}(\zeta)}=\frac{i \pi^{2}}{12}-h^{\prime} \ln \left[1+\mathrm{e}^{i h^{\prime}}\right]+i\left[\operatorname{Li}_{2}\left(1+\mathrm{e}^{i h^{\prime}}\right)+\operatorname{Li}_{2}\left(\mathrm{e}^{i h^{\prime}}\right)\right]-\sin h^{\prime},
$$

where $\operatorname{Li}_{2}(\zeta)=\int_{1}^{\zeta}[\ln (t) /(1-t)] \mathrm{d} t$ is the dilogarithm function defined by Spence's integral (Abramowitz \& Stegun 1972). It is also of interest to note that as $h^{\prime} \rightarrow 0, G_{\mathrm{HR}}$ satisfies $G_{\mathrm{HR}}\left(h^{\prime}\right) \sim 2 h^{\prime 3} / 9-h^{\prime 5} / 225+O\left(h^{\prime 7}\right)$, again suggesting why the matching of the cube of the free-surface slope is appropriate for the thin-film case and also being suggestive as to how the solution form of the lubrication approximation successfully predicts experimental results even at surprisingly large values of $h^{\prime}$ - as the coefficient of the correction to the cubic power is small. Following the procedure of section 2 we find

$$
G_{\mathrm{HR}}\left(\theta_{\text {out }}\right)-G_{\mathrm{HR}}\left(\theta_{\text {in }}\right)=\epsilon\left(\ln [a / \lambda]+Q_{i}\left(\theta_{\text {in }}\right)-Q_{o}\left(\theta_{\text {out }}\right)\right),
$$

which is the result for the spreading rate found by Hocking and Rivers by matching through an intermediate region.

\section{Conclusion}

The slow moving contact line problem is singular in the nondimensional slip length $\lambda$, with a second regular perturbation in the nondimensional contact line speed $|\mathrm{Ca}|$. The principal realisation of this work is to show that it is the truncation of the $C a$-series of solutions at leading order in $\lambda$ that leads to the breakdown of the overlap between outer and inner asymptotic regions. It is for this reason that a separate intermediate region has previously been used as a bridge. However, for Stokes flow equations the significant terms from the infinite $C a$-series of solutions are now determined, and hence matching may proceed directly between inner and outer regions. We note that whilst some of the cases considered are two-dimensional, the work of Hocking \& Rivers (1982) discussed 
in section 4.2 is three-dimensional (axisymmetric), and our general results in section 2 apply in three-dimensional geometries provided the contact line variations occur at much longer lengthscales compared to the microscopic parameters defining the extent of the inner region. Essentially this means that in complex macroscopic geometries, provided the microstructure appears homogeneous in the matching region and on the order of $\lambda$, then the flow in the contact line vicinity may be treated as a two-dimensional problem, with our results thus applicable.

Importantly, we note that including additional macroscopic effects acting at length scales much greater than the microscopic length scale, such as gravity or substrate heterogeneities, do not change the regime where viscous and surface tension forces balance. This is the physical balance of equation (2.7). It is also important to note that a wide range of models are applied to moving contact line problems. Whilst the specific cases discussed in sections 3-4 all use slip to overcome the moving contact line singularity, a range of other physical effects may be incorporated, from disjoining pressures and diffuseinterfaces (de Gennes 1985; Anderson et al. 1998) to shear-thinning fluid and interface formation models (Weidner \& Schwartz 1994; Shikhmurzaev 1997). In particular, asymptotic analysis of liquid-gas (Sibley et al. 2013c,b) and binary fluid (Sibley et al. 2013a) diffuse-interface models, and of the interface formation model (Sibley et al. 2012) has been recently performed.

Provided the additional physics contained within these models is confined to the inner region near the contact line and neglected elsewhere; and that the models contain viscous and capillarity terms, the results of our analysis persist.

Two example cases of singularity-free contact line models beyond simple slip are those of diffuse-interface methods and hybrid models. Firstly, Yue et al. (2010) showed, using numerical computations, that a binary fluid diffuse-interface model can be mapped to the slip model results of Cox (1986), with the effective slip length being the 'diffusion length' of the binary fluid model - related to the interfacial width, mobility and viscosity. Given this equivalence, it underlines the generality of our analysis to a range of models.

Secondly, the hybrid model of Petrov \& Petrov (1992) combines the solution of Cox (1986), but with the microscopic contact angle from the molecular kinetic theory of Blake \& Haynes (1969). The hybrid model again has two regions, but the microscopic contact angle (denoted $\theta_{\text {in }}$ here) is calculated separately from the molecular kinetic theory equations - rather than chosen directly from phenomenological arguments. Our analysis again applies, as it is still relevant to the matching between slip and outer regions.

A further discussion point is about the applicability of this work to both advancing and receding flows. Eggers $(2004,2005)$ determined a critical Capillary number for thin-film receding flows above which a contact line cannot be present, and a liquid film is instead deposited on the solid. A number of recent works have discussed this wetting failure (Chan et al. 2011, 2012; Snoeijer et al. 2008; Vandre et al. 2013), with analytical results being based on this thin-film analysis. That the analysis is performed in the thin-film approximation is important to note for the analysis, as the results rely on a solution of the full inner region equations given by Duffy \& Wilson (1997), which is a parametric solution involving Airy functions. Given this complexity, even in the thin-film case, such a result is not available in general Stokes flow. However, from the discussion in section 3.1 , we may conclude that the viscocapillary balance of equation (2.7) no longer holds for receding flows above the critical capillary number, and which ultimately leads to the breakdown of the overlap region and matching condition. A naive calculation for the 
limit of applicability would be when $\theta_{\text {out }}=0$. From (2.18) this occurs when

$$
C a^{*}=\left[G(0)-G\left(\theta_{\text {in }}\right)\right]\left[-\ln \lambda+\frac{C_{\text {in }}}{f\left(\theta_{\text {in }}\right)}-\frac{C_{\text {out }}}{f(0)}\right]^{-1},
$$

but further analysis is beyond the scope of this work.

In conclusion, we have determined a general direct matching procedure. Through this result we have also justified the previously used direct matching of the cubes of the free-surface slopes in the thin-film regime, provided equation (2.7) is unaffected. For further simplification of the full Stokes flow problem, it would be of interest to combine the analysis here with that of Snoeijer (2006), where a result beyond the lubrication approximation was found, but without performing the full analysis of Cox (1986).

We thank Dr Benjamin D. Goddard, with whom the numerical scheme used to produce the results of figure 1 was developed in a joint project; we also thank Dr Nikos Savva for useful discussions, and acknowledge financial support from ERC Advanced Grant No. 247031 and Imperial College London through a DTG International Studentship.

\section{Appendix A}

Here, we give details of the forced wetting case in the lubrication approximation used to compute figure 1. This extends the analytical results of Eggers (2005) to a further order for both outer and inner asymptotic regions and discusses how the full numerical results for the full problem, and for the inner region, are computed.

\section{A.1. Outer region asymptotics}

From (3.1), the outer region equation in which slip is negligible slip is given by

$$
-C a / h^{2}=h^{\prime \prime \prime}-h^{\prime}+\theta .
$$

For $|C a| \ll 1$, we consider solutions of the form

$$
h=h_{0}+C a h_{1}+C a^{2} h_{2}+O\left(C a^{3}\right),
$$

and find at leading order that

$$
h_{0}^{\prime \prime \prime}=h_{0}^{\prime}-\theta, \quad h_{0}(0)=0, \quad h_{0}^{\prime}(\infty)=\theta,
$$

where the boundary conditions represent zero height of the film at the contact line and that the slope of the film approaches the (imposed) angle of inclination as $x \rightarrow \infty$. The solution is given by

$$
h_{0}=\theta x+\left(\theta-\theta_{\text {out }}\right)\left(\mathrm{e}^{-x}-1\right),
$$

where $\theta_{\text {out }}$ is the 'apparent' contact angle, and is given by $h_{0}^{\prime}(0)=\theta_{\text {out }}$. The equations at higher orders satisfy the form

$$
f_{n}(x)=h_{n}^{\prime \prime \prime}-h_{n}^{\prime}, \quad \text { at } O\left(C a^{n}\right), \quad \text { for } n \in \mathbb{Z}: n \geqslant 1,
$$

where, for our purposes we are only interested in $f_{1}=-1 / h_{0}^{2}$ and $f_{2}=2 h_{1} / h_{0}^{3}$. This equation is solved by

$$
h_{n}(x)=-\frac{\mathrm{e}^{x}}{2} \int_{1}^{\infty} \mathrm{e}^{-t} f_{n}(t) \mathrm{d} t+\int_{1}^{x}(\cosh (t-x)-1) f_{n}(t) \mathrm{d} t+K_{n} \mathrm{e}^{-x}+K_{n, 2} .
$$


We have set the prefactor of the homogeneous solution $\mathrm{e}^{x}$ such that $h_{n}$ does not diverge for $x \rightarrow \infty$ (remember this is for $h_{n}$, where $n \geqslant 1$ ), and the derivative is then given by

$$
h_{n}^{\prime}(x)=-\frac{\mathrm{e}^{x}}{2} \int_{1}^{\infty} e^{-t} f_{n}(t) \mathrm{d} t-\int_{1}^{x} \sinh (t-x) f_{n}(t) \mathrm{d} t-K_{n} \mathrm{e}^{-x} .
$$

The two coefficients $K_{n}$ and $K_{n, 2}$ are defined through the boundary conditions $h_{n}(0)=0$ and $h_{n}(\infty)=0$, which for $n=1$ and $n=2$ are given by

$$
\begin{aligned}
K_{n} & =\int_{1}^{\infty}\left(\frac{e^{-t}}{2}-1\right) f_{n}(t) d t+\int_{0}^{1}(\cosh (t)-1) f_{n}(t) \mathrm{d} t, \\
K_{n, 2} & =\int_{1}^{\infty} f_{n}(t) \mathrm{d} t .
\end{aligned}
$$

The only remaining parameter to compute the outer region solutions $h_{0}, h_{1}$ and $h_{2}$ is $\theta_{\text {out }}$. This may be ultimately obtained through the matching condition (2.18) and the behaviour of $h_{1}^{\prime}(x)$ as $x \rightarrow 0$. From (A 7) we have

$$
\lim _{x \rightarrow 0} h_{1}^{\prime}(x)=\theta_{\text {out }}^{-2}\left[\ln x+C\left(\theta_{\text {out }}\right)\right] \text {, }
$$

where

$$
\frac{C\left(\theta_{\text {out }}\right)}{\theta_{\text {out }}^{2}}=\int_{1}^{\infty}\left(1-\mathrm{e}^{-t}\right) f_{1}(t) \mathrm{d} t+\int_{0}^{1}\left\{\left(1-\mathrm{e}^{-t}\right) f_{1}(t)+\frac{1}{\theta_{\text {out }}^{2} t}\right\} \mathrm{d} t+\frac{1}{\theta_{\text {out }}^{2}}
$$

and then using the results from sections 2 and 3.1, and in particular matching condition (2.18) and equation (3.2), we have that $\theta_{\text {out }}$ is given to $O\left(C a^{2}\right)$ by the implicit equation

$$
\theta_{\text {out }}^{3}-1=3 C a\left[-\ln \lambda+1-C\left(\theta_{\text {out }}\right)\right] .
$$

This has allowed us to compute the leading and first order outer solutions, as found by Eggers (2005), and to further find the second order solution, to be able to plot these results in figure 1.

\section{A.2. Inner region asymptotics}

In the inner region, $h$ and $x$ are on of the order of the slip length $\lambda$, and hence we rescale through

$$
h=\lambda H, \quad x=\lambda \xi
$$

Equation (3.1) for $\lambda \ll 1$ then transforms to

with boundary conditions

$$
-\frac{C a}{H^{2}+H}=H^{\prime \prime \prime}
$$

$$
H(0)=0, \quad H^{\prime}(0)=1, \quad H^{\prime \prime}(\infty)=0 .
$$

Note that for receding contact lines beyond a critical value of $C a$ the third condition in (A 14) cannot be satisfied, and a constant curvature is instead found. In cases where all conditions in (A 14) hold, as for the outer region, the inner solution is approximated by expanding $\mathrm{H}$ in $\mathrm{Ca}$ as

$$
H(\xi)=H_{0}(\xi)+C a H_{1}(\xi)+C a^{2} H_{2}(\xi)+O\left(C a^{3}\right),
$$

which gives the equations

$$
H_{0}^{\prime \prime \prime}=0, \quad H_{1}^{\prime \prime \prime}=\frac{-1}{H_{0}^{2}+H_{0}}, \quad H_{2}^{\prime \prime \prime}=\frac{H_{1}\left(2 H_{0}+1\right)}{H_{0}^{2}\left(H_{0}+1\right)^{2}},
$$


where the boundary conditions are

$$
H_{n}(0)=0, \quad H_{n}^{\prime}(0)=\left\{\begin{array}{ll}
1 & \text { for } n=0 \\
0 & \text { otherwise }
\end{array}\right\}, \quad H_{n}^{\prime \prime}(\infty)=0 .
$$

The first three orders are solved by

$$
\begin{aligned}
H_{0}(\xi)= & \xi \\
H_{1}(\xi)= & \frac{1}{2}(\xi+1)^{2} \ln (\xi+1)-\frac{1}{2} \xi^{2} \ln \xi-\frac{1}{2} \xi \\
H_{2}(\xi)=- & \frac{1}{2}(\xi+1) \ln (\xi+1)[(\xi+2) \ln \xi+2 \xi-1] \\
& +\frac{\xi}{6}\left[6(\xi+1) \ln \xi-\xi \pi^{2}-9\right]-\left(\xi^{2}+\xi+1\right) \operatorname{Li}_{2}(\xi+1),
\end{aligned}
$$

where $\operatorname{Li}_{2}(\zeta)=\int_{1}^{\zeta}[\ln (t) /(1-t)] \mathrm{d} t$. These results give us three orders of the inner region solution, with agreement with the first two found by Eggers (2005), and these are plotted in figure $1(\mathrm{a})$.

\section{A.3. Numerical computations}

We conclude the Appendix by providing details of the numerical results for the full problem, and for the inner region. For the full problem we solve the third order ODE given in equation (3.1) subject to the boundary conditions of

$$
h(0)=0, \quad h^{\prime}(0)=1, \quad h^{\prime}(\infty)=\theta .
$$

The computation for this third order ODE has been solved through implementing a pseudospectral discretisation for the independent variable (Trefethen 2000), and plotted in figure 1.

The full inner region ODE is once again third order and given in (A 13), with boundary conditions (A 14). As the first derivative is singular at infinity, we choose to solve the ODE with the same pseudospectral method, but in the range $\xi \in[0, L]$, where $H^{\prime \prime}(L)=0$. Three curves are plotted in figure $1(\mathrm{~b})$, for $L=\{50,500,5000\}$. Convergence of the behaviour may be seen for large $\xi$ (and hence large $x$ ) to the intermediate (dashed) curve for increasing $L$.

\section{REFERENCES}

Abramowitz, M. \& Stegun, I. A. 1972 Handbook of mathematical functions: with formulas, graphs, and mathematical tables. New York: Dover Publications.

Anderson, D. M., McFadden, G. B. \& Wheeler, A. A. 1998 Diffuse-interface methods in fluid mechanics. Annu. Rev. Fluid Mech. 30, 139-165.

Blake, T. \& Haynes, J. 1969 Kinetics of liquid/liquid displacement. J. Colloid Interface Sci. 30 (3), 421-423.

Bonn, D., Eggers, J., Indekeu, J., Meunier, J. \& Rolley, E. 2009 Wetting and spreading. Rev. Mod. Phys. 81, 739-805.

Chan, T. S., Gueudré, T. \& Snoeijer, J. H. 2011 Maximum speed of dewetting on a fiber. Phys. Fluids 23, 112103.

Chan, T. S., Snoeijer, J. H. \& Eggers, J. 2012 Theory of the forced wetting transition. Phys. Fluids 24, 072104.

Cox, R. G. 1986 The dynamics of the spreading of liquids on a solid surface. Part 1. Viscous flow. J. Fluid Mech. 168, 169-194.

Cox, R. G. 1998 Inertial and viscous effects on dynamic contact angles. J. Fluid Mech. 357, 249-278. 
Ding, H. \& Spelt, P. D. M. 2007 Inertial effects in droplet spreading: a comparison between diffuse-interface and level-set simulations. J. Fluid Mech. 576, 287-296.

Duffy, B. R. \& Wilson, S. K. 1997 A third-order differential equation arising in thin-film flows and relevant to Tanner's law. Appl. Math. Lett. 10 (3), 63-68.

Eggers, J. 2004 Hydrodynamic theory of forced dewetting. Phys. Rev. Lett. 93, 094502.

EGgers, J. 2005 Existence of receding and advancing contact lines. Phys. Fluids 17 (8), 082106.

De Gennes, P. G. 1985 Wetting: statics and dynamics. Rev. Mod. Phys. 57, 827-863.

Hocking, L. M. 1983 The spreading of a thin drop by gravity and capillarity. Q. J. Mech. Appl. Math. 36 (1), 55-69.

Hocking, L. M. \& Rivers, A. D. 1982 The spreading of a drop by capillary action. J. Fluid Mech. 121, 425-442.

Huh, C. \& Scriven, L. E. 1971 Hydrodynamic model of steady movement of a solid / liquid / fluid contact line. J. Colloid Interface Sci. 35 (1), 85-101.

Peters, I., Snoeijer, J. H., Daerr, A. \& Limat, L. 2009 Coexistence of two singularities in dewetting flows: Regularizing the corner tip. Phys. Rev. Lett. 103, 114501.

Petrov, P. \& Petrov, I. 1992 A combined molecular-hydrodynamic approach to wetting kinetics. Langmuir 8 (7), 1762-1767.

Savva, N. \& Kalliadasis, S. 2009 Two-dimensional droplet spreading over topographical substrates. Phys. Fluids 21 (9), 092102.

Savva, N. \& Kalliadasis, S. 2012 Influence of gravity on the spreading of two-dimensional droplets over topographical substrates. J. Eng. Math. 73 (1), 3-16.

Shikhmurzaev, Y. D. 1997 Moving contact lines in liquid/liquid/solid systems. J. Fluid Mech. 334, 211-249.

Sibley, D. N., Nold, A. \& Kalliadasis, S. 2013a Unifying binary fluid diffuse-interface models in the sharp-interface limit. J. Fluid Mech. 736, 5-43.

Sibley, D. N., Nold, A., Savva, N. \& Kalliadasis, S. $2013 b$ The contact line behaviour of solid-liquid-gas diffuse-interface models. Phys. Fluids 25 (9), 092111.

Sibley, D. N., Nold, A., Savva, N. \& Kalliadasis, S. $2013 c$ On the moving contact line singularity: Asymptotics of a diffuse-interface model. Eur. Phys. J. E 36, 26.

Sibley, D. N., Nold, A., Savva, N. \& Kalliadasis, S. 2014 A comparison of slip, disjoining pressure, and interface formation models for contact line motion through asymptotic analysis of thin two-dimensional droplet spreading. J. Eng. Math. Available as Online First, DOI: $10.1007 / \mathrm{s} 10665-014-9702-9$.

Sibley, D. N., SaVva, N. \& Kalliadasis, S. 2012 Slip or not slip? a methodical examination of the interface formation model using two-dimensional droplet spreading on a horizontal planar substrate as a prototype system. Phys. Fluids 24 (8), 082105.

SnoeiJer, J. H. 2006 Free-surface flows with large slopes: Beyond lubrication theory. Phys. Fluids 18 (2), 021701.

Snoeijer, J. H. \& Andreotti, B. 2013 Moving contact lines: Scales, regimes, and dynamical transitions. Annu. Rev. Fluid Mech. 45, 269-292.

Snoeijer, J. H., Ziegler, J., Andreotti, B., Fermigier, M. \& Eggers, J. 2008 Thick films of viscous fluid coating a plate withdrawn from a liquid reservoir. Phys. Rev. Lett. 100, 244502 .

Sui, Y., Ding, H. \& Spelt, P. D. M. 2014 Numerical simulations of flows with moving contact lines. Annu. Rev. Fluid Mech. 46, 97-119.

Trefethen, L. N. 2000 Spectral methods in MATLAB. Philadelphia: SIAM.

Vandre, E., Carvalho, M. S. \& Kumar, S. 2013 On the mechanism of wetting failure during fluid displacement along a moving substrate. Phys. Fluids 25 (10), 102103.

Vellingiri, R., Savva, N. \& Kalliadasis, S. 2011 Droplet spreading on chemically heterogeneous substrates. Phys. Rev. E 84, 036305.

Voinov, O. V. 1976 Hydrodynamics of wetting. Fluid Dyn. 11 (5), 714-721.

Weidner, D. E. \& Schwartz, L. W. 1994 Contact-line motion of shear-thinning liquids. Phys. Fluids 6, 3535-3538.

Yue, P., Zhou, C. \& Feng, J. J. 2010 Sharp-interface limit of the Cahn-Hilliard model for moving contact lines. J. Fluid Mech. 645, 279-294. 